\title{
Seleção de clones de batata-doce resistentes a Meloidogyne incognita raça 1
}

\author{
Aline Marchese(1), Wilson Roberto Maluf(1), Álvaro Carlos Gonçalves Neto(1), \\ Ranoel José de Sousa Gonçalves ${ }^{(1)}$ e Luiz Antonio Augusto Gomes ${ }^{(1)}$
}

\begin{abstract}
(1)Universidade Federal de Lavras, Departamento de Agricultura, Caixa Postal 3037, CEP 37200-000 Lavras, MG. E-mail: alinemarchese@hotmail.com, wrmaluf@ufla.br, alvarocgneto@hotmail.com, ranoelgoncalves@hotmail.com, laagomes@ufla.br
\end{abstract}

Resumo - O objetivo deste trabalho foi selecionar clones de batata-doce (Ipomoea batatas) resistentes à raça 1 de Meloidogyne incognita e avaliar a eficiência do método de seleção empregado, pela estimação dos coeficientes de variação genética e ambiental e das herdabilidades no sentido amplo. Foram utilizados 123 genótipos de batata-doce, entre os quais quatro cultivares comerciais - Brazlândia Rosada, Brazlândia Roxa, Brazlândia Branca e Palmas -, e 119 acessos previamente selecionados no programa de melhoramento vegetal da Universidade Federal de Lavras. O delineamento experimental utilizado foi o de blocos aumentados, com três tratamentos comuns: as cultivares de batata-doce Brazlândia Branca e Palmas, e a cultivar de tomate Santa Clara, suscetível ao nematoide. A classificação dos níveis de resistência foi realizada de acordo com o fator de reprodução do nematoide e o índice de reprodução relativo à cultivar Santa Clara, de tomateiro. A relação entre os coeficientes de variação genética e ambiental e as herdabilidades no sentido amplo foram altas, tanto para o fator de reprodução quanto para o índice de reprodução dos nematoides, o que demonstra a eficiência do método empregado para a seleção de genótipos resistentes. Foram identificados 57 genótipos promissores de batata-doce, resistentes à raça 1 de $M$. incognita, e selecionados para continuar no programa de melhoramento.

Termos para indexação: Ipomoea batatas, fator de reprodução, galhas, índice de reprodução, nematoide, resistência.

\section{Selection of sweetpotato clones resistant to Meloidogyne incognita race 1}

\begin{abstract}
The objective of this work was to select sweetpotato (Ipomoea batatas) resistant clones to Meloidogyne incognita race 1, and to assess the efficiency of the selection method deployed, through the estimation of genetic and environmental coefficients of variation, and broad-sense heritabilities. Genotypes assessed comprised 123 sweetpotato entries altogether, including four commercial cultivars - Brazlândia Rosada, Brazlândia Roxa, Brazlândia Branca, Palmas - and 119 clones previously selected by the Universidade Federal de Lavras sweetpotato breeding program. The experimental setup was a an augmented block design, using three common treatments: the sweetpotato cultivars Brazlândia branca and Palmas, and the nematode-susceptible tomato cultivar Santa Clara. Nematode resistance levels were defined both by the nematode reproduction factor and by the nematode reproduction index relative to tomato cv. Santa Clara. The ratio between genetic and environmental coefficients of variation and the broad-sense heritability estimates were high, for both nematodes reproduction factor and reproduction index, indicating that the selection method deployed was efficient for the selection of resistant genotypes. Fifty-seven sweetpotato clones were identified as resistant to $M$. incognita race 1, and selected to continue in the sweetpotato breeding program.
\end{abstract}

Index terms: Ipomoea batatas, reproduction factor, root-knot, reproduction index, nematode, resistance.

\section{Introdução}

A batata-doce [Ipomoea batatas (L.) Lam.] é uma hortaliça tuberosa rústica, de ampla adaptabilidade, cultivada em praticamente todo o território brasileiro. Apesar de ter importância social e econômica fundamental, ainda é pouco valorizada no Brasil (Silva et al., 2004a; Ritschel et al., 1999). É uma hortaliça com múltiplos usos, e pode ser utilizada na alimentação humana in natura ou processada industrialmente, nas formas de amido, macarrão e farinha (Centro Internacional de la Papa, 2000). Pode, ainda, ser utilizada na alimentação animal (Xianglin, 2004; Centro Internacional de la Papa, 2000) ou como alternativa na produção de etanol biocombustível (Souza, 2006).

Apesar de apresentar elevado potencial produtivo, no Brasil, é comum encontrar baixas produtividades de batata-doce, decorrentes, principalmente, da utilização 
de materiais genéticos obsoletos e degenerados, em sua maioria suscetíveis a pragas e doenças. A degenerescência é favorecida pelo fato de a cultura ser propagada comercialmente por meio de reprodução assexuada, com uso de ramas, o que acentua o problema a cada geração (Kroth et al., 2004). A utilização de tratos culturais de baixa tecnologia também leva a produtividades aquém da mínima desejável (Silva et al., 2004a). Entre os patógenos, o nematoide-das-galhas (Meloidogyne spp.) é considerado determinante para a baixa produtividade brasileira, nessa cultura (Silveira \& Maluf, 1993; Cardoso et al., 2005).

As principais espécies de nematoides que afetam a cultura são o M. javanica (Treub) Chitwood, 1949 e M. incognita (Kofoid \& White) Chitwood, 1949, raças 1, 2, 3 e 4. Embora a cultura seja considerada como "falsa não hospedeira", pois frequentemente não produz o sintoma mais característico do patógeno - galhas produzidas pela oviposição das fêmeas nas raízes -, as raízes secundárias de plantas de batata-doce apresentam grande potencial para acumular altos níveis populacionais de nematoides (Charchar \& Ritschel, 2004). Durante a infecção, as fêmeas penetram nas raízes, principalmente no sistema radicular secundário, e depositam massas de ovos. Dos ovos eclodem as formas juvenis do primeiro estádio, que infectam novas raízes, e se alimentam de metabólitos da planta até atingirem a fase adulta, quando o ciclo se reinicia (Massaroto, 2008).

Exceto em cultivares de elevada suscetibilidade, em que as galhas depreciam qualitativamente as raízes tuberosas, os efeitos sobre a parte comercial não são diretos, pois os nematoides raramente infectam raízes tuberosas. $\mathrm{O}$ efeito indireto dos nematoides sobre a produtividade ocorre pelo prejuízo ao desenvolvimento das raízes, com redução na disponibilidade de água, nutrientes e assimilados para a planta e queda acentuada de produtividade, circunstância em que pode ocorrer a morte da planta (Charchar \& Ritschel, 2004).

A aplicação de nematicidas como método de controle é uma atividade cara e ineficiente. O uso de genótipos resistentes é a principal técnica de controle do patógeno na cultura, por não onerar o custo de produção e não ser agressivo ao meio ambiente (Pinheiro et al., 2009). Diversos trabalhos vêm sendo realizados nos últimos anos com o objetivo de identificar fontes de resistência, seja para uso como cultivares, seja para utilização em programas de melhoramento visando ao desenvolvimento de novas cultivares resistentes (Silveira \& Maluf, 1993; Azevedo, 1995; Silveira et al., 1997; Wanderley \& Santos, 2004). Alguns genótipos podem ser resistentes a uma raça ou a uma espécie específica de nematoide-das-galhas, mas não a outras. As fontes de resistência relatadas são específicas para as diferentes raças de $M$. incognita (Silveira \& Maluf, 1993; Azevedo, 1995; Massaroto, 2008).

O objetivo deste trabalho foi selecionar clones de batata-doce resistentes a $M$. incognita raça 1 , e avaliar a eficiência do método de seleção empregado, pela estimação dos coeficientes de variação genética e ambiental, e das herdabilidades no sentido amplo.

\section{Material e Métodos}

O ensaio foi conduzido nas instalações da Estação Experimental de Hortaliças da HortiAgro Sementes Ltda., Fazenda Palmital, Município de Ijaci, MG, localizado a $21^{\circ} 14^{\prime} 16^{\prime \prime} \mathrm{S}$ e $45^{\circ} 08^{\prime} 00^{\prime \prime} \mathrm{W}$, com altitude média de $918 \mathrm{~m}$.

Em março de 2008, foram semeadas sementes botânicas oriundas de 31 famílias de meio-irmãos de batata-doce, provenientes dos programas de pesquisa da Universidade Federal do Tocantins (Palmas, TO) e da Embrapa Hortaliças (Brasília, DF). As mudas resultantes atingiram um total de 330 plantas, ou genótipos, e foram levadas ao campo para multiplicação de ramas e posterior instalação do experimento do programa de melhoramento da Universidade Federal de Lavras (Lavras, MG). Neste programa de melhoramento, foram selecionados clones com produtividades preliminares próximas ou superiores a 50 toneladas de raízes por hectare, o que resultou num total de 114 acessos, com denominação 2007HSF-xxx-yy, em que xxx = número da família de meio-irmãos, e yy $=$ número do clone selecionado dentro da família.

Esses acessos foram testados quanto à resistência a $M$. incognita raça 1 . No ensaio, foram utilizados 123 genótipos de batata-doce, entre eles, quatro cultivares comerciais - Brazlândia Rosada, Brazlândia Roxa, Brazlândia Branca e Palmas -, os 114 clones de batata-doce 2007HSF-xxx-yy, selecionados na fase descrita anteriormente, e cinco acessos denominados: UFLA-07-12, UFLA-07-31, UFLA-07-43, UFLA-07-49 e UFLA-07-53, selecionados anteriormente quanto a características de interesse comercial. A cultivar 
de tomate Santa Clara foi utilizada como hospedeira padrão do nematoide (Taylor, 1967). As cultivares de batata-doce Brazlândia Branca e Palmas foram utilizadas como testemunhas suscetível (Silveira \& Maluf, 1993) e resistente (Silveira et al., 1997), respectivamente.

O plantio dos materiais foi realizado em bandejas de poliestireno expandido, com 72 células preenchidas com, aproximadamente, $120 \mathrm{~mL}$ do substrato comercial Plantmax (Eucatex, São Paulo, Brasil), por célula. Foram usadas ramas com aproximadamente $20 \mathrm{~cm}$ de comprimento e três a quatro gemas internodais.

A inoculação foi realizada 30 dias após o plantio. Ovos de $M$. incognita raça 1 foram extraídos de plantas de tomateiros suscetíveis (cultivar Santa Clara) previamente inoculadas, conforme técnica desenvolvida por Hussey \& Baker (1973) e descrita por Silva et al. (2004b). Depois de lavadas, as raízes de tomateiro foram picadas e processadas em liquidificador por 30 segundos, em solução de hipoclorito de sódio a $0,5 \%$. Após a trituração, a solução foi passada em peneira de $0,074 \mathrm{~mm}$, colocada sobre uma peneira de $0,028 \mathrm{~mm}$, juntamente com água abundante. Na peneira de $0,074 \mathrm{~mm}$, ficaram retidos os restos de raízes, enquanto na de $0,028 \mathrm{~mm}$ foram coletados os ovos de M. incognita raça 1 , que foram transferidos para um béquer, com o auxílio de uma pisceta com água pura.

Os ovos foram contados com auxílio de estereomicroscópio. Primeiramente, completou-se o volume de água do béquer para $1.000 \mathrm{~mL}$. Em seguida, homogeneizou-se a solução que continha os ovos, os quais foram retirados com auxílio de uma pipeta, em três alíquotas de $1 \mathrm{~mL}$. As alíquotas foram colocadas em câmara de Peters (Southey, 1970) e levadas ao estereomicroscópio para contagem do número de ovos. O número médio de ovos por $\mathrm{mL}$ da solução foi calculado. Para a inoculação das plântulas de batata-doce, foi utilizada a quantidade total de 3.500 ovos por plântula, aplicados a cada célula preenchida com substrato, com uso de seringa de uso veterinário. A viabilidade do inóculo foi avaliada em câmara de eclosão, e alcançou 59\% de ovos viáveis. Dessa forma, a quantidade de ovos viáveis, aplicada por plântula, foi de 2.065 .

O delineamento experimental utilizado foi $\mathrm{o}$ de blocos aumentados. Foram considerados três tratamentos comuns aos 14 blocos utilizados: tomate cultivar Santa Clara, e as cultivares de batata-doce
Brazlândia Branca e Palmas. Além deles, foram utilizados 121 tratamentos regulares, o que resultou em 163 unidades experimentais, com seis plantas cada.

Sessenta dias após a inoculação, as plantas foram retiradas cuidadosamente das bandejas de poliestireno e suas raízes lavadas para extração dos ovos, conforme a metodologia de Hussey \& Baker (1973) descrita por Silva et al. (2004b). As estimativas do número de ovos foram realizadas pela contagem de $1 \mathrm{~mL}$ da suspensão, em câmara de Peters (Southey, 1970), com uso de microscópio estereoscópico. O número total de ovos foi determinado pela extrapolação dos valores obtidos nesta contagem. Esses valores foram submetidos à análise de variância e, com base nas médias, determinaram-se os seguintes parâmetros: fator de reprodutividade $(\mathrm{FR}=$ população final/população inicial de ovos viáveis) e índice de reprodução. O fator de reprodutividade é usado para definir resistência $(\mathrm{FR}<1)$ e suscetibilidade $(\mathrm{FR} \geq 1)$, segundo Oostenbrink (1966).

O índice de reprodução (IR) de $M$. incognita raça 1 foi determinado com uso da reprodução dos nematoides no tomateiro como testemunha padrão $(100 \%) \mathrm{em}$ comparação com os clones de batata-doce, de acordo com a metodologia estabelecida por Taylor (1967). A população final (Pf) encontrada nos genótipos de batata-doce foi dividida pela encontrada no tomateiro, de modo a definir os índices de reprodução. Com base nesses valores, definiram-se os níveis de resistência de cada genótipo de batata-doce à raça 1 de $M$. incognita, de acordo com o seguinte critério de reprodução estabelecido por Taylor (1967): S, planta suscetível, reprodução normal, IR acima de 51\%; LR, levemente resistente, IR de 26 a 50\%; MoR, moderadamente resistente, com IR de 11 a 25\%; MR, muito resistente, IR de 1 a $10 \%$; AR/I, altamente resistente/imune, IR abaixo de $1 \%$.

Os dados coletados foram submetidos à análise de variância, com os recursos do pacote computacional SAS Institute (2001). A partir das esperanças dos quadrados médios das análises de variância, foram estimadas as variâncias genéticas $\left(\sigma_{\mathrm{g}}^{2}\right)$ e ambientais $\left(\sigma_{\mathrm{e}}^{2}\right)$, e a herdabilidade no sentido amplo $\left(\mathrm{h}_{\mathrm{a}}^{2}\right)$ para cada característica, de acordo com o procedimento de Vencovsky \& Barriga (1992), por meio da seguinte expressão:

$$
\mathrm{h}_{\mathrm{a}}^{2}=\sigma_{\mathrm{g}}^{2} /\left(\sigma_{\mathrm{g}}^{2}+\sigma_{\mathrm{e}}^{2}\right) \times 100
$$

Pesq. agropec. bras., Brasília, v.45, n.9, p.997-1004, set. 2010 
Os coeficientes de variação genética $\left(\mathrm{CV}_{\mathrm{g}}\right)$ e ambiental $\left(\mathrm{CV}_{\mathrm{e}}\right)$, bem como o índice $\mathrm{b}\left(\mathrm{CV}_{\mathrm{g}} / \mathrm{CV}_{\mathrm{e}}\right)$, para as características avaliadas, foram estimados a partir das seguintes expressões:

$$
\mathrm{CV}_{\mathrm{g}}(\%)=\left(\left(\left(\sigma_{\mathrm{g}}^{2}\right)^{0,5}\right) / \mu\right) \times 100, \text { e } C V_{\mathrm{e}}=\left(\left(\left(\sigma_{\mathrm{e}}^{2}\right)^{0,5}\right) / \mu\right) \times 100
$$

\section{Resultados e Discussão}

Houve efeito significativo dos genótipos de batata-doce sobre o fator de reprodução (FR) e índice de reprodução (IR) de $M$. incognita raça 1 , o que indica que deve haver variabilidade genética entre eles (Tabela 1). Os coeficientes de variação ambiental foram altos para ambas as características. Porém, coeficientes dessas magnitudes são comuns em experimentos com batata-doce (Azevedo et al., 2000; Cardoso et al., 2005). Contudo, estimativas bem mais elevadas do coeficiente de variação genética foram observadas, tanto para o fator de reprodução como para o índice de reprodução, o que indica, além de alta variabilidade entre os materiais, uma situação bastante favorável à seleção de clones resistentes.

As estimativas de herdabilidade no sentido amplo foram altas, e refletem o fato de que a maior parte da variabilidade fenotípica foi de natureza genética, e indicam que a seleção baseada nessas características poderia ser realizada com eficiência. Isto é reforçado pelos valores do índice $\mathrm{b}\left(\mathrm{CV}_{\mathrm{g}} / \mathrm{CV}_{\mathrm{e}}\right)$, que foram superiores a 1,0, o que também indica uma situação favorável à seleção.

Tabela 1. Resumo da análise de variância para o fator de reprodução (FR) e índice de reprodução (IR) de Meloidogyne incognita em raízes de clones de batata-doce.

\begin{tabular}{lccc}
\hline Fontes de variação & GL & Fator de reprodução & Índice de reprodução \\
\hline Tratamento & 122 & $76,58^{* *}$ & $10.756,49^{* *}$ \\
T x C & 1 & $72,78^{\text {ns }}$ & $8.646,70^{\text {ns }}$ \\
Clones (C) & 120 & $98,34^{* *}$ & $8.492,91^{* *}$ \\
Testemunhas (T) & 2 & $11,7087^{* *}$ & $1.466,25^{* *}$ \\
Erro & 26 & 17,95 & $3.144,79$ \\
\hline Média & & 5,89 & 74,69 \\
\hline CVe (\%) & 71,85 & 75,08 \\
CVg (\%) & 132,33 & 128,33 \\
$\mathrm{~b}=\mathrm{CVg} / \mathrm{CVe}$ & & 1,84 & 1,71 \\
$\mathrm{~h}_{a}^{2}(\%)$ & 74,63 & 68,87 \\
\hline
\end{tabular}

CVe, coeficiente de variação ambiental; CVg, coeficiente de variação genético; índice $\mathrm{b}, \mathrm{CVg} / \mathrm{CVe}$; $\mathrm{e}_{\mathrm{a}}^{2}$, herdabilidade no sentido amplo. nsNão significativo. * e **Significativo a $5 \%$ e $1 \%$ de probabilidade, respectivamente, pelo teste de $\mathrm{F}$.
Segundo o critério citado por Oostenbrink (1966), $54,03 \%$ dos genótipos testados foram considerados suscetíveis, com fator de reprodução igual ou acima de 1,0 (Tabela 2). Entre os genótipos suscetíveis, incluem-se as cultivares comerciais Brazlândia Rosada, Brazlândia Branca e tomate cultivar Santa Clara, resultados que estão de acordo com Silveira \& Maluf (1993) e Charchar \& Ritschel (2004). Os genótipos 2007HSF001-16, 2007HSF020-12, 2007HSF025-04 e 2007HSF028-11 foram os que apresentaram as maiores médias para o fator de reprodução, com valores muito superiores à média apresentada pela testemunha suscetível Brazlândia Branca. Nesses materiais, a população de nematoides chegou a crescer mais de 30 vezes relativamente à população inicial de inóculo.

Pelo mesmo critério de Oostenbrink (1966), foram classificados como resistentes $45,97 \%$ dos genótipos testados, com FR abaixo de 1,0 (Tabela 2). Esta alta frequência de materiais resistentes à raça 1 de M. incognita era esperada, uma vez que Azevedo (1995), ao testar a resistência de clones de batata-doce ao $M$. javanica e às raças $1,2,3$ e 4 de $M$. incognita, obteve um grande número de materiais resistentes ao $M$. javanica e às raças 1,3 e 4 de $M$. incognita e verificou maior dificuldade de seleção para resistência à raça 2 de $M$. incognita .

Os clones UFLA-07-43, 2007HSF001-09 e 2007HSF002-14 apresentaram as menores médias para fator de reprodução, indicativo de alta resistência ao nematoide. O material denominado UFLA-07 é um genótipo já testado em fases avançadas do programa de melhoramento da Ufla, e possui alta produtividade potencial e características de interesse comercial que, somadas à resistência ao nematoide $M$. incognita raça 1 , o torna promissor para o mercado. As cultivares comerciais Brazlândia Roxa e Palmas, classificadas como resistentes por Silveira et al. (1997), Charchar \& Ritschel (2004) e Massaroto (2008), também foram identificadas como padrões de resistência segundo o critério de Oostenbrink (1966).

Segundo o critério de reprodução estabelecido por Taylor (1967), foram constatados desde genótipos suscetíveis até altamente resistentes ou imunes de acordo com seu índice de reprodução (Tabela 3). Entre os genótipos testados, 33,07\% foram classificados como suscetíveis, incluindo as cultivares de batatadoce, Brazlândia Rosada e Brazlândia Branca, utilizadas como testemunhas, e a cultivar de tomate 
cultivar Santa Clara; 10,48\% foram classificados como levemente resistentes, $10,48 \%$ moderadamente resistentes, $40,32 \%$ muito resistentes, incluindo as testemunhas Palmas e Brazlândia Roxa, e 5,65\% altamente resistentes ou imunes. Genótipos resistentes de pimentão (Peixoto et al., 1999), feijão-comum e feijão-vagem (Ferreira et al., 2010) foram obtidos com sucesso ao se utilizar o tomate como hospedeiro padrão, segundo esse critério, o que demonstra sua efetividade.

Os genótipos classificados pelo índice de reprodução como muito resistentes e altamente resistentes

Tabela 2. Média do fator de reprodução (FR) de Meloidogyne incognita raça 1 nos 123 genótipos de batata-doce avaliados e na cultivar de tomate Santa Clara, e classificação desses genótipos quanto a resistência (R) e suscetibilidade (S) ao nematoide ${ }^{(1)}$.

\begin{tabular}{|c|c|c|c|c|c|c|c|c|}
\hline Genótipo & FR & Classe & Genótipo & FR & Classe & Genótipo & FR & Classe \\
\hline 2007HSF001-01 & 0,64 & $\mathrm{R}$ & 2007HSF007-26 & 3,37 & $\mathrm{~S}$ & 2007HSF022-10 & 0,28 & $\mathrm{R}$ \\
\hline 2007HSF001-09 & 0,04 & $\mathrm{R}$ & 2007HSF007-27 & 0,23 & $\mathrm{R}$ & 2007HSF022-12 & 2,88 & S \\
\hline 2007HSF001-16 & 37,35 & S & 2007HSF009-06 & 0,19 & $\mathrm{R}$ & 2007HSF022-16 & 1,49 & S \\
\hline 2007HSF001-17 & 1,49 & S & 2007HSF010-01 & 9,52 & S & 2007HSF022-19 & 0,68 & $\mathrm{R}$ \\
\hline 2007HSF001-21 & 0,14 & $\mathrm{R}$ & 2007HSF010-06 & 5,03 & S & 2007HSF023-08 & 0,57 & $\mathrm{R}$ \\
\hline 2007HSF001-22 & 0,31 & $\mathrm{R}$ & 2007HSF010-08 & 3,84 & S & 2007HSF024-01 & 0,29 & $\mathrm{R}$ \\
\hline 2007HSF001-23 & 0,58 & $\mathrm{R}$ & 2007HSF010-12 & 11,87 & S & 2007HSF024-02 & 13,06 & S \\
\hline 2007HSF001-24 & 0,07 & $\mathrm{R}$ & 2007HSF010-17 & 11,87 & S & 2007HSF024-04 & 22,62 & S \\
\hline 2007HSF001-25 & 0,35 & $\mathrm{R}$ & 2007HSF010-23 & 2,30 & S & 2007HSF024-06 & 1,00 & S \\
\hline 2007HSF001-26 & 0,09 & $\mathrm{R}$ & 2007HSF010-25 & 0,82 & $\mathrm{R}$ & 2007HSF025-04 & 32,90 & S \\
\hline 2007HSF001-28 & 0,45 & $\mathrm{R}$ & 2007HSF010-30 & 1,16 & $\mathrm{~S}$ & 2007HSF026-01 & 7,41 & S \\
\hline 2007HSF001-37 & 0,15 & $\mathrm{R}$ & 2007HSF010-31 & 16,47 & S & 2007HSF026-02 & 7,36 & S \\
\hline 2007HSF001-40 & 10,13 & S & 2007HSF010-33 & 0,17 & $\mathrm{R}$ & 2007HSF026-05 & 3,50 & S \\
\hline 2007HSF001-41 & 0,07 & $\mathrm{R}$ & 2007HSF010-35 & 5,16 & $\mathrm{~S}$ & 2007HSF027-05 & 0,71 & $\mathrm{R}$ \\
\hline 2007HSF001-45 & 0,62 & $\mathrm{R}$ & 2007HSF010-37 & 1,00 & $\mathrm{~S}$ & 2007HSF027-07 & 1,47 & S \\
\hline 2007HSF001-47 & 0,46 & $\mathrm{R}$ & 2007HSF010-41 & 16,87 & S & 2007HSF027-08 & 0,19 & $\mathrm{R}$ \\
\hline 2007HSF001-58 & 0,11 & $\mathrm{R}$ & 2007HSF010-47 & 1,03 & S & 2007HSF027-09 & 6,57 & S \\
\hline 2007HSF002-02 & 0,07 & $\mathrm{R}$ & 2007HSF011-01 & 12,50 & S & 2007HSF027-10 & 4,84 & S \\
\hline 2007HSF002-04 & 0,23 & $\mathrm{R}$ & 2007HSF011-02 & 10,89 & S & 2007HSF027-12 & 27,51 & S \\
\hline 2007HSF002-05 & 3,39 & S & 2007HSF011-05 & 14,38 & $\mathrm{~S}$ & 2007HSF027-16 & 14,90 & S \\
\hline 2007HSF002-08 & 0,14 & $\mathrm{R}$ & 2007HSF011-06 & 0,34 & $\mathrm{R}$ & 2007HSF028-05 & 18,06 & S \\
\hline 2007HSF002-10 & 0,41 & $\mathrm{R}$ & 2007HSF011-10 & 23,28 & $\mathrm{~S}$ & 2007HSF028-06 & 0,08 & $\mathrm{R}$ \\
\hline 2007HSF002-11 & 0,14 & $\mathrm{R}$ & 2007HSF012-02 & 0,16 & $\mathrm{R}$ & 2007HSF028-08 & 3,82 & S \\
\hline 2007HSF002-14 & 0,06 & $\mathrm{R}$ & 2007HSF013-03 & 1,40 & $\mathrm{~S}$ & 2007HSF028-11 & 31,30 & S \\
\hline 2007HSF002-16 & 19,29 & S & 2007HSF013-04 & 10,63 & S & 2007HSF028-16 & 0,56 & $\mathrm{R}$ \\
\hline 2007HSF002-19 & 0,39 & $\mathrm{R}$ & 2007HSF014-04 & 0,73 & $\mathrm{R}$ & 2007HSF029-01 & 0,18 & $\mathrm{R}$ \\
\hline 2007HSF004-03 & 2,77 & S & 2007HSF014-05 & 0,40 & $\mathrm{R}$ & 2007HSF029-02 & 6,69 & S \\
\hline 2007HSF004-04 & 19,86 & S & 2007HSF016-05 & 21,18 & $\mathrm{~S}$ & 2007HSF029-03 & 3,10 & S \\
\hline 2007HSF004-06 & 1,93 & S & 2007HSF018-03 & 7,36 & $\mathrm{~S}$ & 2007HSF029-09 & 2,92 & S \\
\hline 2007HSF004-08 & 0,30 & $\mathrm{R}$ & 2007HSF019-01 & 0,15 & $\mathrm{R}$ & 2007HSF030-02 & 0,87 & $\mathrm{R}$ \\
\hline 2007HSF005-06 & 28,55 & S & 2007HSF020-05 & 1,73 & S & 2007HSF030-10 & 0,34 & $\mathrm{R}$ \\
\hline 2007HSF006-13 & 0,50 & $\mathrm{R}$ & 2007HSF020-07 & 0,38 & $\mathrm{R}$ & 2007HSF031-04 & 0,18 & $\mathrm{R}$ \\
\hline 2007HSF006-16 & 12,32 & S & 2007HSF020-08 & 0,611 & $\mathrm{R}$ & UFLA-07-12 & 1,35 & S \\
\hline 2007HSF006-17 & 3,84 & S & 2007HSF020-12 & 33,80 & S & UFLA-07-31 & 0,35 & $\mathrm{R}$ \\
\hline 2007HSF007-04 & 8,89 & S & 2007HSF021-01 & 2,28 & $\mathrm{~S}$ & UFLA-07-43 & 0,02 & $\mathrm{R}$ \\
\hline 2007HSF007-10 & 0,24 & $\mathrm{R}$ & 2007HSF022-02 & 0,11 & $\mathrm{R}$ & UFLA-07-49 & 0,30 & $\mathrm{R}$ \\
\hline 2007HSF007-15 & 4,02 & S & 2007HSF022-03 & 0,09 & $\mathrm{R}$ & UFLA-07-53 & 0,18 & $\mathrm{R}$ \\
\hline 2007HSF007-16 & 4,86 & S & 2007HSF022-04 & 1,49 & S & Brazlândia Branca & 17,43 & S \\
\hline 2007HSF007-17 & 8,17 & S & 2007HSF022-05 & 0,84 & $\mathrm{R}$ & Brazlândia Rosada & 9,68 & $\mathrm{~S}$ \\
\hline 2007HSF007-18 & 6,30 & S & 2007HSF022-06 & 15,95 & $\mathrm{~S}$ & Brazlândia Roxa & 0,36 & $\mathrm{R}$ \\
\hline 2007HSF007-21 & 0,86 & $\mathrm{R}$ & 2007HSF022-09 & 1,87 & $\mathrm{~S}$ & Palmas & 0,13 & $\mathrm{R}$ \\
\hline
\end{tabular}

${ }^{(1)}$ Diferença mínima significativa (DMS) entre tratamentos regulares do mesmo bloco, 30,25; DMS entre tratamentos regulares de blocos diferentes, 34,91; DMS entre tratamentos comuns, 8,06; DMS entre tratamentos comuns e regulares, 25,20 , segundo teste de Tukey a $5 \%$ de probabilidade. 
ou imunes representaram 45,97\% dos genótipos avaliados (Tabela 3). Estes genótipos foram os mesmos classificados como resistentes com base no fator de reprodução. Dentro dessa perspectiva, foram selecionados como resistentes 57 clones para dar continuidade ao programa de melhoramento.

$\mathrm{Na}$ comparação dos dois critérios, observa-se coerência, e ambos são eficientes para a identificação e

Tabela 3. Média dos índice de reprodução (IR) de Meloidogyne incognita raça 1 e classificação quanto à resistência ${ }^{(1)}$ de 123 genótipos de batata-doce e a cultivar de tomate Santa Clara, usada como testemunha padrão de susceptibilidade ${ }^{(2)}$.

\begin{tabular}{|c|c|c|c|c|c|c|c|c|}
\hline Genótipo & IR (\%) & Classe & Genótipo & IR (\%) & Classe & Genótipo & IR (\%) & Classe \\
\hline 2007HSF001-01 & 7,59 & MR & 2007HSF007-26 & 39,83 & LR & 2007HSF022-10 & 3,34 & MR \\
\hline 2007HSF001-09 & 0,48 & $\mathrm{AR} / \mathrm{I}$ & 2007HSF007-27 & 2,77 & MR & 2007HSF022-12 & 34,10 & LR \\
\hline 2007HSF001-16 & 442,02 & $\mathrm{~S}$ & 2007HSF009-06 & 2,29 & MR & 2007HSF022-16 & 17,57 & MoR \\
\hline 2007HSF001-17 & 17,57 & MoR & 2007HSF010-01 & 112,70 & $\mathrm{~S}$ & 2007HSF022-19 & 8,02 & MR \\
\hline 2007HSF001-21 & 1,62 & MR & 2007HSF010-06 & 59,50 & $\mathrm{~S}$ & 2007HSF023-08 & 6,78 & MR \\
\hline 2007HSF001-22 & 3,63 & MR & 2007HSF010-08 & 45,37 & LR & 2007HSF024-01 & 3,44 & MR \\
\hline 2007HSF001-23 & 6,88 & MR & 2007HSF010-12 & 140,50 & $\mathrm{~S}$ & 2007HSF024-02 & 154,54 & S \\
\hline 2007HSF001-24 & 0,86 & $\mathrm{AR} / \mathrm{I}$ & 2007HSF010-17 & 140,50 & $\mathrm{~S}$ & 2007HSF024-04 & 267,72 & $\mathrm{~S}$ \\
\hline 2007HSF001-25 & 4,11 & MR & 2007HSF010-23 & 27,22 & LR & 2007HSF024-06 & 11,84 & MoR \\
\hline 2007HSF001-26 & 1,05 & MR & 2007HSF010-25 & 9,65 & MR & 2007HSF025-04 & 389,30 & $\mathrm{~S}$ \\
\hline 2007HSF001-28 & 5,35 & MR & 2007HSF010-30 & 13,75 & MoR & 2007HSF026-01 & 87,68 & S \\
\hline 2007HSF001-37 & 1,72 & MR & 2007HSF010-31 & 194,94 & $\mathrm{~S}$ & 2007HSF026-02 & 87,11 & $\mathrm{~S}$ \\
\hline 2007HSF001-40 & 119,89 & $\mathrm{~S}$ & 2007HSF010-33 & 2,01 & MR & 2007HSF026-05 & 41,45 & LR \\
\hline 2007HSF001-41 & 0,86 & $\mathrm{AR} / \mathrm{I}$ & 2007HSF010-35 & 61,03 & $\mathrm{~S}$ & 2007HSF027-05 & 8,40 & MR \\
\hline 2007HSF001-45 & 7,35 & MR & 2007HSF010-37 & 11,84 & MoR & 2007HSF027-07 & 17,38 & MoR \\
\hline 2007HSF001-47 & 5,44 & MR & 2007HSF010-41 & 199,62 & $\mathrm{~S}$ & 2007HSF027-08 & 2,29 & MR \\
\hline 2007HSF001-58 & 1,26 & MR & 2007HSF010-47 & 12,23 & MoR & 2007HSF027-09 & 77,75 & S \\
\hline 2007HSF002-02 & 0,76 & $\mathrm{AR} / \mathrm{I}$ & 2007HSF011-01 & 147,95 & $\mathrm{~S}$ & 2007HSF027-10 & 57,21 & $\mathrm{~S}$ \\
\hline 2007HSF002-04 & 2,67 & MR & 2007HSF011-02 & 128,84 & $\mathrm{~S}$ & 2007HSF027-12 & 325,50 & $\mathrm{~S}$ \\
\hline 2007HSF002-05 & 40,11 & LR & 2007HSF011-05 & 170,20 & $\mathrm{~S}$ & 2007HSF027-16 & 176,31 & $\mathrm{~S}$ \\
\hline 2007HSF002-08 & 1,60 & MR & 2007HSF011-06 & 4,01 & MR & 2007HSF028-05 & 213,75 & $\mathrm{~S}$ \\
\hline 2007HSF002-10 & 4,87 & MR & 2007HSF011-10 & 275,45 & $\mathrm{~S}$ & 2007HSF028-06 & 0,96 & $\mathrm{AR} / \mathrm{I}$ \\
\hline 2007HSF002-11 & 1,62 & MR & 2007HSF012-02 & 1,91 & MR & 2007HSF028-08 & 45,18 & LR \\
\hline 2007HSF002-14 & 0,67 & $\mathrm{AR} / \mathrm{I}$ & 2007HSF013-03 & 16,52 & MoR & 2007HSF028-11 & 370,32 & S \\
\hline 2007HSF002-16 & 228,27 & $\mathrm{~S}$ & 2007HSF013-04 & 125,85 & $\mathrm{~S}$ & 2007HSF028-16 & 6,59 & MR \\
\hline 2007HSF002-19 & 4,58 & MR & 2007HSF014-04 & 8,60 & MR & 2007HSF029-01 & 2,10 & MR \\
\hline 2007HSF004-03 & 32,76 & LR & 2007HSF014-05 & 4,78 & MR & 2007HSF029-02 & 79,18 & $\mathrm{~S}$ \\
\hline 2007HSF004-04 & 235,05 & $\mathrm{~S}$ & 2007HSF016-05 & 250,62 & $\mathrm{~S}$ & 2007HSF029-03 & 36,68 & LR \\
\hline 2007HSF004-06 & 22,83 & MoR & 2007HSF018-03 & 87,11 & S & 2007HSF029-09 & 34,57 & LR \\
\hline 2007HSF004-08 & 3,53 & MR & 2007HSF019-01 & 1,81 & MR & 2007HSF030-02 & 10,32 & MR \\
\hline 2007HSF005-06 & 337,82 & $\mathrm{~S}$ & 2007HSF020-05 & 20,44 & MoR & 2007HSF030-10 & 4,01 & MR \\
\hline 2007HSF006-13 & 5,92 & MR & 2007HSF020-07 & 4,49 & MR & 2007HSF031-04 & 2,10 & MR \\
\hline 2007HSF006-16 & 145,75 & $\mathrm{~S}$ & 2007HSF020-08 & 7,16 & MR & UFLA-07-12 & 15,95 & MoR \\
\hline 2007HSF006-17 & 45,46 & LR & 2007HSF020-12 & 400,00 & $\mathrm{~S}$ & UFLA-07-31 & 4,15 & MR \\
\hline 2007HSF007-04 & 105,16 & $\mathrm{~S}$ & 2007HSF021-01 & 26,93 & LR & UFLA-07-43 & 0,29 & $\mathrm{AR} / \mathrm{I}$ \\
\hline 2007HSF007-10 & 2,87 & MR & 2007HSF022-02 & 1,34 & MR & UFLA-07-49 & 3,53 & MR \\
\hline 2007HSF007-15 & 47,56 & LR & 2007HSF022-03 & 1,05 & MR & UFLA-07-53 & 2,10 & MR \\
\hline 2007HSF007-16 & 57,50 & $\mathrm{~S}$ & 2007HSF022-04 & 17,57 & MoR & Brazlândia qBranca & 206,27 & $\mathrm{~S}$ \\
\hline 2007HSF007-17 & 96,66 & $\mathrm{~S}$ & 2007HSF022-05 & 9,93 & MR & Brazlândia Rosada & 114,52 & $\mathrm{~S}$ \\
\hline 2007HSF007-18 & 74,50 & $\mathrm{~S}$ & 2007HSF022-06 & 188,73 & $\mathrm{~S}$ & Brazlândia Roxa & 4,30 & MR \\
\hline 2007HSF007-21 & 10,22 & MR & 2007HSF022-09 & 22,16 & MoR & Palmas & 1,64 & MR \\
\hline
\end{tabular}

${ }^{(1)}$ S, suscetível (reprodução normal), IR acima de 51\%; LR, levemente resistente, IR de 26 a 50\%; MoR, moderadamente resistente, IR de 11 a $25 \%$; MR, muito resistente, IR de $1 \%$ a $10 \%$; AR/I, altamente resistente/imune, IR abaixo de $1 \%$. ${ }^{(2)}$ Diferença mínima significativa (DMS) entre tratamentos regulares do mesmo bloco, 400,40; DMS entre tratamentos regulares de blocos diferentes, 462,36; DMS entre tratamentos comuns, 107,01; DMS entre tratamentos comuns e regulares, 332,71, segundo teste de Tukey, a 5\% de probabilidade. 
seleção de materiais genéticos com resistência à raça 1 do $M$. incognita. Porém, observa-se que, em razão do índice de reprodução proporcionar maior distribuição de classes distintas - I/AR, MR, MoR, LR e S -, é possível maior flexibilidade para estabelecer um ponto de truncagem dos genótipos a serem selecionados como resistentes.

$\mathrm{Na}$ sequência do programa de melhoramento de batata-doce da Universidade Federal de Lavras, os clones selecionados deverão ter sua resistência testada para outras raças e espécies de nematoides, uma vez que há ausência de correlação entre os níveis de resistência das diferentes raças e espécies de nematoides causadores de galhas (Silveira \& Maluf, 1993; Charchar \& Ritschel, 2004).

\section{Conclusões}

1. A relação entre o coeficiente de variação genética e ambiental e a herdabilidade no sentido amplo são altas tanto para o fator de reprodução quanto para o índice de reprodução, o que demonstra a eficiência do método empregado na seleção de genótipos resistentes.

2. Entre os clones de batata-doce avaliados, $45,97 \%$ são resistentes à raça 1 de Meloidogyne incognita.

\section{Agradecimentos}

À Fundação de Amparo à Pesquisa de Minas Gerais, ao Conselho Nacional de Desenvolvimento Científico e Tecnológico, à Coordenação de Aperfeiçoamento Pessoal de Nível Superior, à Universidade Federal de Lavras, à Fundação de Apoio ao Ensino, Pesquisa e Extensão, à Fundação de Desenvolvimento Científico e Cultural e à Empresa Hortiagro Sementes, pelo apoio.

\section{Referências}

AZEVEDO, S.M. de Avaliação de famílias de meios-irmãos de batata-doce [Ipomoea batatas (L.) Lam.] quanto à resistência aos nematóides do gênero Meloidogyne e aos insetos de solo. 1995. 61p. Dissertação (Mestrado) - Universidade Federal de Lavras, Lavras.

AZEVEDO, S.M. de; FREITAS, J.A.; MALUF, W.R.; SILVEIRA, M.A. da. Desempenho de clones e métodos de plantio de batata-doce. Acta Scientiarum, v.22, p.901-905, 2000.

CARDOSO, A.D.; VIANA, A.E.S.; RAMOS, P.A.S.; MATSUMOTO, S.N.; AMARAL, C.L.F.; SEDIYAMA, T.; MORAIS, O.M. Avaliação de clones de batata-doce em Vitória da Conquista. Horticultura Brasileira, v.23, p.911-914, 2005.
CENTRO INTERNACIONAL DE LA PAPA. CIP sweetpotato facts. Quito: CIP, 2000. Disponível em: <http://cipotato.org/ sweetpotato/facts/facts.asp $>$. Acesso em: 8 set 2010.

CHARCHAR, J.M.; RITSCHEL, P.S. Avaliação do banco de germoplasma de batata-doce da Embrapa Hortaliças para resistência a Meloidogyne spp. Brasília: Embrapa Hortaliças, 2004. 28p. (Embrapa Hortaliças. Boletim de pesquisa e desenvolvimento, 3).

FERREIRA, S.; GOMES, L.A.A.; MALUF, W.R.; CAMPOS, V.P.; CARVALHO FILHO, J.L.S. de; SANTOS, D.C. Resistance of Dry bean and Snap bean Cultivars to Root-knot nematodes. Hortiscience, v.45, p.320-322, 2010.

HUSSEY, R.S.; BARKER, K.R. A comparison of methods of collecting inocula of Meloidogyne spp. including a new technique. Plant Disease Reporter, v.57, p.1025-1028, 1973.

KROTH, L.L.; DANIELS, J.; PIEROBOM, C.R. Degenerescência da batata-doce no Rio Grande do Sul. Revista Brasileira de Agrociência, v.10, p.79-82, 2004.

MASSAROTO, J.A. Características agronômicas e produção de silagem de clones de batata doce. 2008. 73p. Tese (Doutorado) Universidade Federal de Lavras, Lavras.

OOSTENBRINK, M. Major characteristics of the relation between nematodes and plants. Mededelingen Van De landbouwhogeschool Te Wageningen, v.66, p.1-46, 1966.

PEIXOTO, J.R.; MALUF, W.R.; CAMPOS, V.P. Avaliação de linhagens, híbridos $F_{1}$ e cultivares de pimentão quanto à resistência a Meloidogyne spp ${ }^{1}$. Pesquisa Agropecuária Brasileira, v.34, p.2259-2265, 1999.

PINHEIRO, J.B.; BOITEUX, L.S.; LOPES, C.A.; SILVA, G.O. da. Identificação de fontes de resistência ao nematóide Meloidogyne mayaguensis em acessos de tomateiro (Solanum Lycopersicon). Brasília: Embrapa Hortaliças, 2009. 19p. (Embrapa Hortaliças. Boletim de pesquisa e desenvolvimento, 56).

RITSCHEL, P.S.; LOPES, C.A.; HUAMÁN, Z.; FERREIRA, M.E.; FRANÇA, F.H.; MENEZES, J.E.; TEIXEIRA, D.M.C.; TORRES, A.C.; CHARCHAR, J.M.; THOMAZELLI, L. Organização do banco ativo de germoplasma de batata-doce: situação atual e perspectivas. In: QUEIROZ, M.A. de; GOEDERT, C.O.; RAMOS, S.R.R. (Ed.). Recursos genéticos e melhoramento de plantas para o Nordeste Brasileiro. Petrolina: Embrapa-CPATSA; Brasília: Embrapa-Cenargen, 1999. Disponível em: <http://www. cpatsa.embrapa.br/catalogo/livrorg/batatadoce.pdf $>$. Acesso em: 17 nov 2009.

SAS INSTITUTE. SAS/STAT software: changes and enhancements. Release 8. Cary: SAS Institute, 2001.

SILVA, J.B.C. da; LOPES, C.A.; MAGALHÃES, J.S. Cultura da batata doce. Brasília: Embrapa Hortaliças, 2004a. (Embrapa Hortaliças. Sistemas de produção, 6). Disponível em: <http://www. cnph.embrapa.br/sistprod/batatadoce/index.htm>. Acesso em: 12 dez. 2009.

SILVA, L.H.C.P.; CAMPOS, J.R.; DUTRA, M.R.; CAMPOS, V.P. Aumento da resistência de cultivares de tomate a Meloidogyne incognita com aplicação de acibenzolar-S-metil. Nematologia Brasileira, v.28, p.199-206, 2004b. 
SILVEIRA, M.A.; AZEVEDO, S.M.; MALUF, W.R.; CAMPOS, V.P.; MOMENTÉ, V.G. Palmas e Canuanã: novas cultivares de batata-doce resistentes aos nematóides do gênero Meloidogyne. Horticultura Brasileira, v.15, p.122-123, 1997.

SILVEIRA, M.A.; MALUF, W.R. Resistência de clones de batata-doce à Meloidogyne spp. Horticultura Brasileira, v.11, p.131-133, 1993.

SOUTHEY, J.F. Laboratory methods for work with plant and soil nematodes. 5.ed. London: Ministry of Agriculture Fisheries and Food, 1970. 148p.

SOUZA, F.R. Estabelecimento de um processo fermentativo utilizando células livres, a partir de clones de batata-doce [Ipomoea batatas (L.) Lam.] selecionadas para as condições de Palmas-TO. 2006. 85p. Dissertação (Mestrado) - Universidade Federal do Tocantins, Palmas.
TAYLOR, A.L. Introduction to research on plant nematology: an FAO guide to study and control of the plant-parasitic nematodes. Rome: Food And Agricultural Organization of the United Nations, 1967. 133p.

VENCOVSKY, R.; BARRIGA, P. Genética biométrica no fitomelhoramento. Ribeirão Preto: Sociedade Brasileira de Genética, 1992. 486p.

WANDERLEY, M.J.A.; SANTOS, J.M. Resistência de cultivares de batata-doce a Meloidogyne incognita. Fitopatologia Brasileira, v.29, p.437-440, 2004.

XIANGLIN, L. The livestock revolution and feed demand in China. In: FUGLIE, K.O.; HERMANN, M. (Ed.). Sweetpotato post-harvest research and development in China. Bogor: International Potato Center, 2004. p.40-47.

Recebido em 29 de março de 2010 e aprovado em 2 de agosto de 2010 